assessed by mRSS, may not directly correlate with its effect on patients' health related quality of life (HRQoL). The Scleroderma Skin Patientreported Outcome (SSPRO) was specifically developed to assess the skin-related HRQoL in SSc. No skin-specific PRO in SSc has been prospectively validated in a clinical trial.

Objectives: Validate the SSPRO prospectively in a clinical trial.

Methods: The SSPRO, Patient Global Assessment (PtGA), Physician Global Assessment (MDGA), HAQ-DI, mRSS, FVC\% predicted, PROMIS29 questionnaire, and ACR CRISS were assessed prospectively in a Phase 2 study of lenabasum in dcSSc. SSPRO has 18 items that assess four SSc skin-related HRQoL domains. The Phase 2 study of lenabasum had a 4-month double-blinded portion ( $N=41$ completers) followed by an open-label extension ( $\mathrm{N}=38$ entered). Spearman correlations of baseline values and change values were determined for SSPRO and other outcome measures. Mean change in SSPRO scores were determined in subjects with increasing levels of improvement in other efficacy outcomes.

Results: At baseline, SSPRO correlated moderately with all other outcome measures except for $\mathrm{FVC} \%$, as expected, with strongest correlations with PtGA, HAQ-DI, and PROMIS-29 pain interference and social role domains (Table 1). The mean change in SSPRO scores at 3 and 12 months correlated mostly moderately ( $r=0.25$ to 0.62$)$ with the mean change in other outcome scores, but correlations were low or inconsistent with mRSS, FVC\%, and PROMIS-29 anxiety domain (Table 1). Significance of correlations in some outcomes was hampered by small magnitudes of change.

Mean SSPRO generally increased in subjects with increasing levels of improvement in HAQ-DI, PtGA, mRSS, and ACR CRISS, although less consistently with mRSS and ACR CRISS at 3 months (Table 2).

Conclusion: SSPRO score correlates with both physician and patientreported outcomes at baseline, though correlations with the latter were stronger. Change in SSPRO reflects changes in how the patient feels (PtGA, PROMIS-29 pain interference and depression domains) and functions (HAQ-DI, PROMI-29 physical function and social role domains) and less consistently with physician-assessed outcomes. Subjects with improvement $>$ MID levels of -0.250 in HAQ-DI, -1 in PtGA, and -5 in mRSS had SSPRO improvements of -22 to -23 points at 12 months.

Disclosure of Interests: Ada Man Consultant for: Advisory Board Fee: Abbvie, Janssen, Boeringer Ingelheim, Nancy Dgetluck Employee of: Corbus Pharmaceuticals, Inc., Brian Conley Employee of: Corbus Pharmaceuticals, Inc., Barbara White Shareholder of: Corbus Pharmaceuticals, Inc., Employee of: Corbus Pharmaceuticals, Inc.

DOI: 10.1136/annrheumdis-2019-eular.3388

\section{FRI0335 PROGNOSTIC IMPACT AND CLINICAL CHARACTERISTICS OF INTERSTITIAL PNEUMONIA WITH AUTOIMMUNE FEATURES IN A MULTIDISCIPLINARY SETTING}

Emiliano Marasco ${ }^{1}$, Federica Meloni ${ }^{2}$, Giovanni Zanframundo ${ }^{1}$, Adele Valentini ${ }^{3}$, Valentina Morandi ${ }^{1}$, Veronica Codullo ${ }^{1}$, Lorenzo Volpiano ${ }^{2}$, Claudia La Carruba ${ }^{2}$ Francesca Motta ${ }^{1}$, Carlomaurizio Montecucco ${ }^{1}$, Lorenzo Cavagna ${ }^{1}$.

${ }^{1}$ Rheumatology, University and IRCCS Policlinico S. Matteo Foundation, Pavia, Italy; ${ }^{2}$ Pneumology, University and IRCCS Policlinico S. Matteo Foundation, Pavia, Italy; ${ }^{3}$ Institute of Radiology, Fondazione IRCCS Policlinico San Matteo, Pavia, Italy

Background: Interstitial Pneumonia with Autoimmune feature (IPAF) is a recently defined disease that includes Interstitial Lung Disease (ILD) patients with features of autoimmunity, not satisfying any of the established classification criteria for connective tissue diseases (CTD). Even if IPAF pateints share clinical and serological findings with CTD, we are unaware about the clinical evolution of IPAF pateints.

Objectives: To define the characteristics and evolution of IPAF patients in a multidisciplinary setting.

Methods: We selected patients with a diagnosis of IPAF referring to a multidisciplinary Rheumatology/Pneumology/Radiology team at our hospital. We excluded from the analysis patients positive for antisynthetase antibodies, as diagnosed with antisynthetase syndrome. Data were retrospectively collected from our hospital medical records.

Results: We analyzed 25 patients (19 females, 76\%, 6 males, 24\%), with a median onset age of 67 years (interquartile range, IQR, 59-74) and a follow-up of 32 months (IQR 22-69). ANA test was positive in $23(92 \%)$ cases (fig 1), whereas cytoplasmic positivity was observed in 17 (68\%). Anti-ENA screen was positive in 9 patients $(36 \%)$ ( 7 [28\%] anti-Ro52 and $2[8 \%]$ anti-RNP). One patient (4\%) was positive for anti-PM-Scl, 1 (4\%) for anti-Mi2 and 1 (4\%) for anti-Ku antibodies. These 2 latter antibodies are not included in the serological domain of IPAF. Patients had mainly a Non Specific Interstitial Pneumonia (NSIP) pattern (19 [76\%]; fibrosing in 7 [28\%], and with concomitant organizing pneumonia (OP) in $3[12 \%])$. Three (12\%) patients had Usual Interstitial Pneumonia-like and $3(12 \%)$ an OP pattern. The majority of patients (15 [60\%]) satisfied only the morphological and serological domains. Clinical domains satisfied were: arthritis (4 [16\%]), Raynaud's phenomenon (5 [20\%]), palmar telangiectasias (2 [8\%]), mechanic's hands (1 [4\%]) and hiker's feet (1 [4\%]). We observed other findings not included in IPAF criteria but suggestive for CTDs, both clinical (inflammatory myopathy $3[12 \%]$; dry eye1 $[4 \%]$; sclerodactyly $1[4 \%]$ ), and instrumental (scleroderma pattern at naifold capillaroscopy 1 [4\%]; dilated esophagus at barium X-rays 2 [8\%]). Clinical spectrum time course was variable in $5(20 \%)$ cases: $3(12 \%)$ patients developed arthritis after ILD, and $2(8 \%)$ developed ILD respec-

Table 1. Spearman Correlations for SSPRO and Other Efficacy Outcomes at Baseline and for Change in SSPRO and Other Efficacy Outcomes at 3 and 12 months ( $r$, P-value)

\begin{tabular}{|c|c|c|c|c|c|c|c|c|c|c|c|}
\hline \multirow[t]{2}{*}{ Time } & \multirow[t]{2}{*}{ PtGA } & \multirow[t]{2}{*}{ MDGA } & \multirow[t]{2}{*}{ HAQ-DI } & \multirow[t]{2}{*}{ mRSS } & \multirow[t]{2}{*}{ FVC, $\%$} & \multirow{2}{*}{$\begin{array}{c}\text { ACR } \\
\text { CRISS }\end{array}$} & \multicolumn{5}{|c|}{ PROMIS-29 Domain } \\
\hline & & & & & & & $\begin{array}{l}\text { Pain } \\
\text { Interference }\end{array}$ & Social Role & $\begin{array}{l}\text { Physical } \\
\text { Function }\end{array}$ & Depression & Anxiety \\
\hline Baseline & 0.61 & 0.51 & 0.52 & 0.44 & -0.07 & & $0.62<0.0001$ & -0.570 .0001 & -0.330 .034 & 0.510 .0007 & 0.41 \\
\hline & $<0.0001$ & 0.0007 & 0.0005 & 0.004 & 0.670 & & & & & & 0.008 \\
\hline Change 3 months & 0.260 .124 & 0.300 .067 & 0.290 .083 & $\begin{array}{c}0.02 \\
0.932\end{array}$ & $\begin{array}{l}-0.12 \\
0.483\end{array}$ & -0.200 .235 & 0.460 .005 & -0.400 .015 & -0.460 .004 & 0.330 .044 & $\begin{array}{c}0.14 \\
0.395\end{array}$ \\
\hline $\begin{array}{l}\text { Change } 12 \\
\text { months }\end{array}$ & 0.280 .103 & 0.150 .394 & 0.250 .145 & $\begin{array}{l}0.20 \\
0.251\end{array}$ & $\begin{array}{l}-0.53 \\
0.001\end{array}$ & -0.480 .004 & 0.410 .014 & $\begin{array}{l}-0.62< \\
0.0001\end{array}$ & -0.250 .152 & 0.270 .119 & $\begin{array}{c}0.21 \\
0.234\end{array}$ \\
\hline
\end{tabular}

Table 2. Mean Change in SSPRO Associated with Increasing Levels of Improvement in Other Outcomes

\begin{tabular}{|c|c|c|c|c|c|c|c|c|c|c|c|c|c|c|c|}
\hline $\begin{array}{l}\text { Efficacy } \\
\text { Outcome }\end{array}$ & \multicolumn{4}{|c|}{ HAQ-DI improvement } & \multicolumn{3}{|c|}{ PtGA improvement } & \multicolumn{4}{|c|}{ mRSS improvement } & \multicolumn{4}{|c|}{ 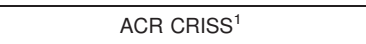 } \\
\hline $\begin{array}{l}\text { Improvement } \\
\text { at least }\end{array}$ & None & -0.125 & -0.250 & -0.500 & None & -1 & -2 & None & -3 & -5 & -8 & $\begin{array}{c}< \\
0.30\end{array}$ & $\begin{array}{l}\geq \\
0.30\end{array}$ & $\begin{array}{c}\geq \\
0.60\end{array}$ & $\begin{array}{c}\geq \\
0.90\end{array}$ \\
\hline $\begin{array}{l}3 \text { months } \\
(\mathrm{N}=37)\end{array}$ & -9.9 & -17.6 & -20.2 & -22.5 & -11.9 & -15.3 & -16.5 & -11.0 & -14.7 & -16.3 & -10.3 & -10.2 & -17.8 & -17.1 & -14.5 \\
\hline 12 months & -17.9 & -20.2 & -22.7 & -27.2 & -16.5 & -21.9 & -21.5 & -11.6 & -19.6 & -23.2 & -23.1 & -13.0 & -23.5 & -26.6 & -27.3 \\
\hline
\end{tabular}

${ }^{1}$ ACR CRISS score is absolute, not change, score. 
tively after arthritis and inflammatory myopathy onset. Three (12\%) patients were admitted to the Intensive Care Unit for Rapidly Progressive (RP) ILD and 2 died (respectively 2 months and 54 months after ILD onset), whereas the alive patient had 2 ICU admission for RP-ILD. Five $(20 \%)$ patients, including the only 1 dismissed from ICU, needed home $\mathrm{O}_{2}$ therapy. Ongoing and previous therapies are reported in figure 2.

Conclusion: We showed that the prognosis of IPAF is highly variable, with patients experiencing RP ILD, other slow progressive worsening of respiratory functions and other a substantially stable disease. Furthermore, we showed other findings, laboratory, clinical and instrumental, that could help clinicians in a better identification and stratification of IPAF patients. As a matter of fact, at present, IPAF appears as a generic term including very different conditions that can be further differentiated according to clinical and serological data.

\section{REFERENCES:}

[1] Fischer A, et al. Eur Respir J 2015

[2] Sciré CA, et al. Resp Med 2017

[3] Sambataro G, et al. Eur Resp Rev 2018

[4] Cavagna L, et al. Eur Resp Rev 2018

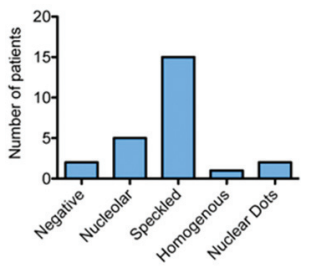

Figure 1. Antinuclear antibodies determination results

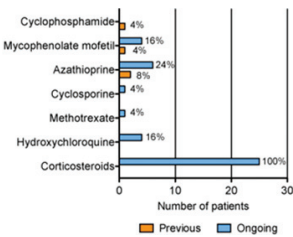

Figure 2. Ongoing (at last follow-up) and previous treatments

Disclosure of Interests: Emiliano Marasco: None declared, Federica Meloni: None declared, Giovanni Zanframundo: None declared, Adele Valentini: None declared, Valentina Morandi: None declared, Veronica Codullo: None declared, Lorenzo Volpiano: None declared, Claudia La Carruba: None declared, Francesca Motta: None declared, Carlomaurizio Montecucco Speakers bureau: AbbVie, Bristol-Myers Squibb, Celgene, Sanofi, Genzyme, Lilly, MSD, Pfizer, UCB, Lorenzo Cavagna: None declared DOI: 10.1136/annrheumdis-2019-eular.2704

\section{FRI0336 INCIDENCE OF IDIOPATHIC INFLAMMATORY MYOPATHIES (IIM) IN ADULTS IN FIFE, SCOTLAND, UK}

John McLaren ${ }^{1}$, Paul Allcoat ${ }^{1}$, Michael Hearst ${ }^{1}$, Elizabeth Furrie ${ }^{2}$, Sarah Hailwood ${ }^{1} .{ }^{1} N H S$ Fife, Fife Rheumatic Diseases Unit, Kirkcaldy, United Kingdom; ${ }^{2} \mathrm{NHS}$ Tayside, Immunology Laboratory Service, Dundee, United Kingdom

\section{Background:}

Objectives: To determine the Adult incidence of IIM in the Kingdom of Fife, Scotland. The Fife population is 370,000 and constitutes $6.8 \%$ of the Scottish Population (5,425,000). 98\% of the Fife population is Caucasian. We also sought to characterise the incident IIM cases in terms of subtype and autoantibody profile.

Methods: All newly diagnosed Adult cases ( $\geq 18$ years) of IIM between 01.03.17 and 30.11.18 were recorded (11.75 year period). The cases were identified by a search of the Fife Rheumatic Diseases Unit Database using IIM-specific ICD-10 codes. These were cross-referenced with a prospective personal database of IIM cases established by the Lead Author (JMcL) on 26.02.17. In addition a search of all Fife patients enrolled in the UKMYONET study was also performed. All patients with 'definite' or 'probable' IIM by the Bohan \& Peter 1977 criteria; Clinically defined Inclusion Body Myositis (IBM) by the ENMC 2011 criteria; or other specific criteria in the case of Clinically Amyopathic Dermatomyositis (CADM) and Immune-mediated Necrotising myopathy (IMNM) were included.

Results: 53 newly diagnosed Adult IIM cases were identified. 11 Dermatomyositis (DM) (3 NXP2; 2 Jo-1; 2 TIF1-G; 1 Mi-2; 3 Myositis-specific antibody (MSA) not tested). 4 Polymositis (PM) (1 Jo-1; 1 MDA5; 1 MSA/Myosits-associated antibody (MAA) negative; 1 MSA not tested). 12 Anti-synthetase syndrome (8 Jo-1; 3 PL7; 1 PL12) and 3 patients with Jo-1 isolated Interstitial lung disease (ILD). 5 Overlap Connective tissue disease (CTD) : 2 Systemic Lupus Erythematosus; 2 Systemic sclerosis; 1 Mixed CTD. 5 Cancer-associated myositis (CAM) : 2 lung (1 NXP2 $\mathrm{DM}$ \& 1 SRP PM); 1 ovarian (DM, MSA not tested); 1 Breast (DM, TIF1-G); 1 Prostate (DM, Mi-2). 5 CADM (2 MDA5; 1 TIF1-G; 1 NXP2 1 MSA not tested). 4 IMNM (2 HMGCR; 1 HMGCR overlap with IBM; 1 SRP overlap with IBM who developed bladder cancer $<3$ years after diagnosis). 4 sporadic IBM (2 cN-1A/Mup44 of which 1 overlap with Primary Sjogren's Syndrome; 2 MAA not tested).

Conclusion: The incidence of IIM in Fife, Scotland is 4.5 cases per year which equates to an annual incidence of 12.2 cases per million population. This is comparable with the incidence data published for Salford in the UK (17.6). However the IIM subtypes differed from Salford in that Fife has a much lower incidence of PM. 40/49 (82\%) of Fife patients who were tested were found to be MSA positive.

Disclosure of Interests: None declared

DOI: 10.1136/annrheumdis-2019-eular.8242

\section{FRI0337 INCIDENCE AND PREVALENCE OF MYOSITIS ASSESSED BY MULTI-SOURCES CAPTURE- RECAPTURE METHODOLOGY}

alain meyer ${ }^{1}$, Léa Debrut ${ }^{2}$, Lionel Spielmann ${ }^{3}$, Gilles Blaison ${ }^{3}$, Pierre Kieffer ${ }^{4}$ Thierry Martin ${ }^{1}$, Jean Sibilia ${ }^{1}$. ${ }^{1}$ Hôpitaux Universitaires de Strasbourg, Strasbourg, France; ${ }^{2}$ Université de Strasbourg, Strasbourg, France; ${ }^{3}$ Hôpitaux de Colmar, Colmar, France; ${ }^{4}$ Hôpitaux de Mulhouse, Mulhouse, France

Background: Precise epidemiology of myositis epidemiology remains largely unknown (1). Surveys based solely on administrative claims benefit from large case ascertainment but may be influenced by miscoding and misdiagnosing. The use of medical records with charts review benefit from accurate diagnosis but exhaustive ascertainment is difficult to achieve because numerous specialists are involved and cases may concern both inpatients and outpatients.

To overcome these difficulties we undertook a capture-recapture survey that takes advantage of a multi-sources case ascertainment to estimate the number of cases missed by any one source and to correct the prevalence rate (2).

Objectives: To assess the incidence and prevalence of myositis in Alsace, a region of eastern France.

Methods: Alsace, region of eastern France, is home to about 2 million inhabitants benefiting from high access to healthcare and a labialized referral center for myositis. Seeking care outside is uneasy because of peculiar geography. Myositis patients were retrieved through three separate sources: i) all general practitioners and community specialist ii) Muscle pathology center records, iii) all public and private hospitals records, vi) all public and private laboratory records. Incident and prevalent cases fulfilling the ACR/EULAR criteria for myositis were included.

Results: The responses to the questionnaires sent to the physicians $(n=3452)$, yielded 105 potential myositis cases. All hospital centres contacted $(n=13)$ participated in the study and 1335 potential myositis patients were recorded by this source. 263 potential myositis cases were identified through muscle pathology center records. 13 laboratories participate in the studies and 324 potential myositis patients were recorded by this source. We thus received 1863 records of suspected myositis after excluding duplicates within each sources. The thorough review of the corresponding medical charts is currently ongoing and at this stage $10 \%$ of the potential cases fulfilled the ACR/EULAR criteria for myositis.

Conclusion: This first study based on a multi-sources capture-recapture methodology and ACR/EULAR criteria is very likely to provide an accurate estimation of myositis epidemiology.

\section{REFERENCES:}

[1] Incidence and prevalence of inflammatory myopathies: a systematic review.

[2] Meyer A, Meyer N, Schaeffer M, Gottenberg JE, Geny B, Sibilia J.

[3] Rheumatology (Oxford). 2015;54:50-63.

[4] Brief Report: Spatial Heterogeneity of Systemic Sclerosis in France: High Prevalence in the Northeast Region.

[5] Meyer A, Chifflot H, Chatelus E, Kleinmann JF, Ronde-Ousteau C, Klein D, Jégu J, Geny B, Hirshi S, Canuet M, Blaison G, Kieffer P, Lipsker D, 